\title{
Single-center Experience of 24 Cases of Tailgut Cyst
}

\author{
Ahmad Sakr ${ }^{1,2}$, Ho Seung Kim ${ }^{1}$, Yoon Dae $\operatorname{Han}^{1}$, Min Soo Cho ${ }^{1}$, Hyuk Hur ${ }^{1}$, Byung Soh Min ${ }^{1}$, \\ Kang Young Lee ${ }^{1}$, Nam Kyu Kim ${ }^{1}$ \\ ${ }^{1}$ Division of Colorectal Surgery, Department of Surgery, Severance Hospital, Yonsei University College of Medicine, Seoul, Korea; ${ }^{2}$ Colorectal \\ Surgery Unit, Department of General Surgery, Mansoura Faculty of Medicine, Mansoura University Hospitals, Mansoura, Egypt
}

Purpose: Tailgut cysts are rare congenital or developmental lesions that arise from vestiges of the embryological hindgut. They are usually present in the presacral space. We report our single-center experience with managing tailgut cysts. Methods: We conducted a retrospective analysis of 24 patients with tailgut cyst treated surgically at the Colorectal Surgery Department of Severance Hospital, Yonsei University, Seoul, South Korea, between 2007-2018.

Results: This study included 24 patients (18 females) with a median age of 51.5 years (range, 21-68 years). Ten cases were symptomatic and 14 were asymptomatic. Cysts were retrorectal in 21 patients. Cysts were below the coccyx level in 16 patients, opposite the coccyx in 6 , and above the coccyx in 2. Cysts were supralevator in 5 patients, had a supra- and infralevator extension in 18 patients, and were infralevator in 1 . Ten patients were managed using an anterior laparoscopic approach, 11 using a posterior approach, and 3 using a combined approach. Mean cyst size was $5.5 \pm 2.7 \mathrm{~cm}$. Postoperative complications were Clavien-Dindo (CD) classification grade II in 9 patients (37.5\%) and CD grade III in 1 (4.2\%). The posterior approach group showed the highest rate of complications $(\mathrm{P}=0.021)$. Patients managed using a combined approach showed a larger cyst size $(\mathrm{P}<0.001)$, longer operation times $(\mathrm{P}<0.001)$, and a greater likelihood of tumor level above the coccyx $(\mathrm{P}=0.002)$ compared to other approaches. The tumors of 2 male patients were malignant: 1 was a neuroendocrine tumor treated with radiotherapy, while the other was a closely followed adenocarcinoma. Median follow-up was 12 months (range, 1-66 months) with no recurrence.

Conclusion: Tailgut cysts are uncommon but can cause perineal or pelvic pain. Complete surgical excision via an appropriate approach according to tumor size, location, and correlation with adjacent pelvic floor muscles is the key treatment.

Keywords: Tailgut cyst; Presacral tumors; Retrorectal space

\section{INTRODUCTION}

Presacral tailgut cysts (retrorectal cystic hamartomas) are rare congenital lesions that are believed to arise from remnants of the hindgut when incomplete involution occurs during embryogenesis $[1,2]$. These cysts are usually present in the presacral space, which is anteriorly bound by the rectum, posteriorly by the lower

Received: October 23, 2018 - Accepted: December 18, 2018

Correspondence to: Nam Kyu Kim, M.D.

Division of Colorectal Surgery, Department of Surgery, Yonsei University

College of Medicine, 50-1 Yonsei-ro, Seodaemun-gu, Seoul 03722, Korea

Tel: +82-2-2228-2100, Fax: +82-2-313-8289

E-mail:namkyuk@yuhs.ac

ORCID code: https://orcid.org/0000-0003-0639-5632

(C) 2019 The Korean Society of Coloproctology

This is an open-access article distributed under the terms of the Creative Commons Attribution NonCommercial License (http://creativecommons.org/licenses/by-nc/4.0) which permits unrestricted noncommercial use, distribution, and reproduction in any medium, provided the original work is properly cited. sacrum and coccyx, superiorly by the peritoneal reflection, inferiorly by the levator ani and pelvic floor, and laterally by the iliac vessels and ureters $[3,4]$. However, cysts also occur in the prerectal, perianal, and perirenal regions [5-7]. These lesions are rare and can present diagnostic and management challenges. Most studies are case reports and case series [5, 8-12]. Surgical treatment is always required because of complications including malignant transformation, infection, and perianal fistula formation. The aim of this study was to report our single-center experience with tailgut cysts, including their management and surgical outcomes.

\section{METHODS}

This retrospective case series included 24 patients with a pathologically confirmed tailgut cyst treated between January 2007 and August 2018 at the Colorectal Surgery Department of Severance 
Volume 35, Number 5, 2019

Hospital, Yonsei University, Seoul, South Korea. The study protocol was approved by the Institutional Review Board of Yonsei University (approval number: 4-2018-0862). Written informed consents were waived off because of being retrospective study.

Patients' medical records were reviewed for demographic variables, history, clinical presentation, previous surgical history, diagnostic methods, operative details, complications, histopathology, follow-up, and recurrence rates. All patients were diagnosed using pelvic magnetic resonance imaging (MRI) (Fig. 1).

Anterior (transabdominal), posterior (paracoccygeal), and combined (anterior + posterior) approaches were used. Most of the tumors were below the coccygeal level, so the posterior approach was usually used. However, the anterior approach was sometimes sufficient for removing tumors at the coccygeal level. For prerectal cysts, the anterior approach was used.

For the anterior approach, laparoscopy was used for all patients to enable complete mobilization of the rectum through the medial, lateral, posterior, deep posterior, and anterior dissection until the cyst was separated from the rectum for retrorectal cysts. For prerectal cysts in female patients, the aim was to mobilize the cyst by separating the rectum from the vagina, then completely excising the cyst via a small mini-laparotomy (Fig. 2).

For the posterior approach (Fig. 3), patients were placed in a prone jack knife position and a straight paracoccygeal or inverted Y-shaped incision was usually made to just before the anal sphinc-
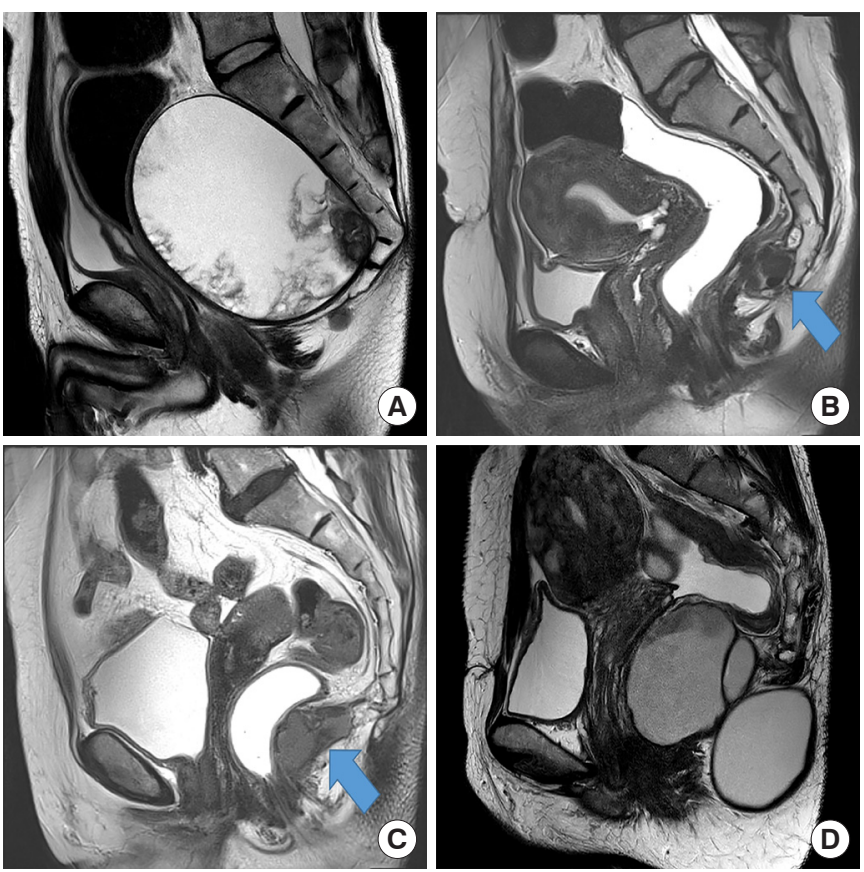

Fig. 1. (A) Malignant cyst extending above the coccyx showing soft tissue component inside. (B) The arrow pointing at the cyst opposite the level of the coccyx. (C) The arrow pointing at the cyst below the level of the coccyx. (D) Dumbbell shaped cyst. ter. Cysts were dissected with care taken to avoid perforating the rectum or cyst or leaving any cyst remnant. A digital rectal examination was performed intraoperatively to ensure rectal integrity. Large cysts at the coccyx or lower sacrum (S4-5) were removed with the help of orthopedic surgeons. All wounds were closed over negative suction drains.

For larger cysts, a combined approach was used starting with the laparoscopic anterior approach. After wound closure, patients were moved into the prone jack knife position for the posterior approach.

The choice of surgical approach was made according to tumor size and correlation with the pelvic floor. For infralevator tumors, the posterior approach was sufficient. For supralevator tumors, the anterior approach was necessary. For cysts with infralevator and supralevator extensions, if small $(<5 \mathrm{~cm})$, the posterior ap-

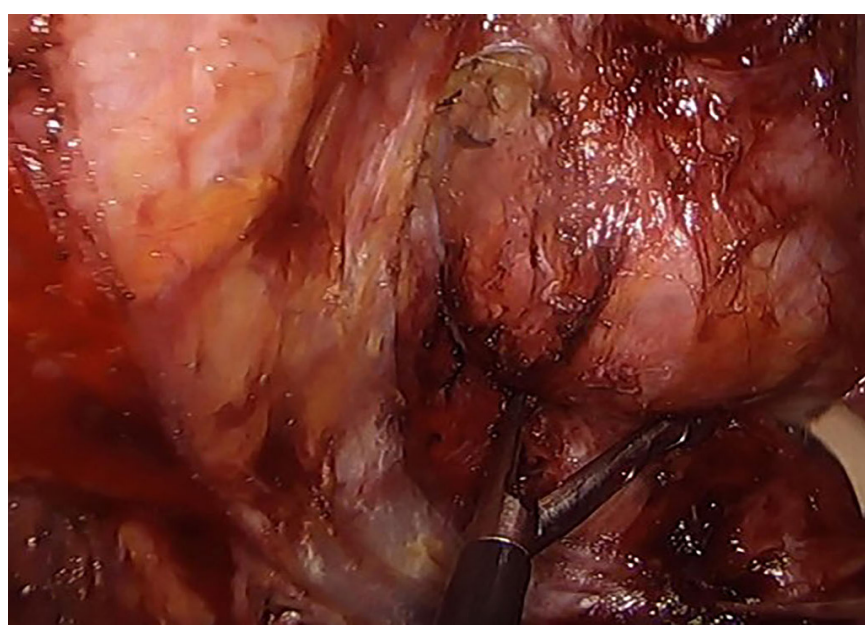

Fig. 2. Laparoscopic anterior approach view.

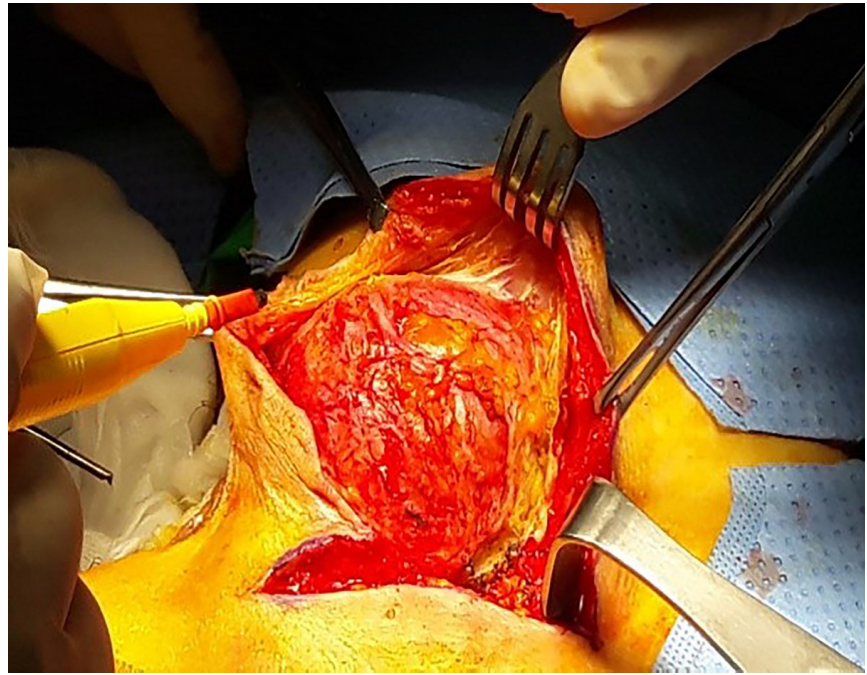

Fig. 3. Posterior approach view. 


\section{Coloproctology

proach was sufficient. When cysts were $5-10 \mathrm{~cm}$, the anterior approach was advised. Cysts $>10 \mathrm{~cm}$, may be multilocular with a dumbbell shape, so the combined approach was used.

\section{Data analysis}

Statistical analysis was accomplished with IBM SPSS Statistics ver. 23.0 (IBM Co., Armonk, NY, USA). Quantitative data were described as mean \pm standard deviation. Qualitative data were presented as frequencies and proportions. Chi-square and 1-way analysis of variance tests were used. P-values $<0.05$ were considered significant.

\section{RESULTS}

Our study included 24 patients who underwent surgical treat-

Table 1. Patient demographics

\begin{tabular}{|c|c|}
\hline Characteristic & Value \\
\hline Age (yr) & $51.5(21.0-68.0)$ \\
\hline \multicolumn{2}{|l|}{ Sex } \\
\hline Male & $6(25.0)$ \\
\hline Female & $18(75.0)$ \\
\hline \multicolumn{2}{|l|}{ Symptoms } \\
\hline Asymptomatic & $14(58.3)$ \\
\hline Lower abdominal pain & $4(16.7)$ \\
\hline Perineal pain & $3(12.5)$ \\
\hline Palpable perianal swelling & $1(4.2)$ \\
\hline Perianal abscess & $1(4.2)$ \\
\hline Sacrococcygeal sinus & $1(4.2)$ \\
\hline Mass size (cm) & $5.2(2.3-14.0)$ \\
\hline \multicolumn{2}{|l|}{ Tumor location } \\
\hline Prerectal & $3(12.5)$ \\
\hline Retrorectal & 21 (87.5) \\
\hline \multicolumn{2}{|l|}{ Tumor level related to coccyx } \\
\hline Below & $16(66.7)$ \\
\hline Opposite & $6(25.0)$ \\
\hline Above & $2(8.3)$ \\
\hline \multicolumn{2}{|c|}{ Tumor level related to pelvic floor } \\
\hline Supralevator & $5(20.8)$ \\
\hline Supra- + infralevator & $18(75.0)$ \\
\hline Infralevator & $1(4.2)$ \\
\hline \multicolumn{2}{|l|}{ Approach } \\
\hline Anterior, laparoscopic & $10(41.7)$ \\
\hline Posterior & $11(45.8)$ \\
\hline Combined & $3(12.5)$ \\
\hline
\end{tabular}

(Continued to the next) ment for tailgut cyst that aimed for complete removal (Table 1). The median patient age was 51.5 years (range, 21-68 years). Of the patients 18 (75\%) were females. Clinical presentations included pelvic pain in 4 patients, perineal pain in 3, palpable perianal swelling in 2, and a sacrococcygeal sinus with pus discharge posteriorly on the lower back in 1 patient. In the other 14 asymptomatic patients, cysts were incidentally discovered on radiology: 2 for urology problems; 4 for abdominal pain; 2 for chronic cholecystitis; 2 for gynecology problems; 4 via a medical checkup; and 2 via a colonoscopy, of which one was a rectal submucosal tumor.

For the 3 prerectal cysts, 1 was at the rectouterine pouch, 1 was at the rectovaginal septum, while the last one was in a male patient anterior to the rectum. The 3 prerectal tumors were below the level of the coccyx; all were at the supralevator level and managed via an anterior laparoscopic approach. An intraoperative vaginal injury occurred and was repaired in the 2 female patients. In the male patient, a rectal perforation occurred, for which repair and a loop ileostomy were performed. Postoperative courses were uneventful and patients were discharged home safely. The ileostomy was closed after 6 months in the male patient. Among the other 21 retrorectal cases, on MRI, tumors were below the level of

Table 1. Continued

\begin{tabular}{|c|c|}
\hline Characteristic & Value \\
\hline \multicolumn{2}{|l|}{ Intraoperative complication } \\
\hline Vaginal injury & $2(8.3)$ \\
\hline Rectal serosal injury & $2(8.3)$ \\
\hline Rectal injury requiring diversion & $2(8.3)$ \\
\hline \multicolumn{2}{|l|}{ Postoperative complication } \\
\hline Wound seroma and infection & $7(29.2)$ \\
\hline Fluid collection in ischiorectal fossa & $1(4.2)$ \\
\hline Lower limb weakness, pelvic floor dyssynergia & $1(4.2)$ \\
\hline Sexual dysfunction & $1(4.2)$ \\
\hline \multicolumn{2}{|l|}{ Clavien-Dindo classification grade } \\
\hline I & $0(0)$ \\
\hline$\|$ & $9(37.5)$ \\
\hline III & $1(4.2)$ \\
\hline IV & $0(0)$ \\
\hline V & $0(0)$ \\
\hline \multicolumn{2}{|l|}{ Pathology } \\
\hline Benign & $22(91.7)$ \\
\hline Malignanta ${ }^{\mathrm{a}}$ & $2(8.3)$ \\
\hline Follow-up (mo) & $12(1-66)$ \\
\hline Recurrence & $0(0)$ \\
\hline Mortality & $0(0)$ \\
\hline
\end{tabular}

Values are presented as median (range) or number (\%).

ane adenocarcinoma with close follow-up, 1 carcinoid with radiotherapy. 
the coccyx (coccyx down) in 13 and at the level of the coccyx in 5. Seven were managed using the anterior laparoscopic approach and most were supra- and infralevator. One female patient experienced a rectal perforation intraoperatively for whom ileostomy was performed. Her postoperative course was good, and she underwent ileostomy closure 2 months later. The posterior approach was used for the other 11 patients, who had tumors that mostly extended to the supralevator and infralevator levels. The other 3 patients ( 2 females, 1 male), on MRI, 2 had cysts extending above the level of the coccyx to the sacral promontory and 1 had a cyst opposite the coccyx; all were managed via the combined approach because of the large cyst size $(10-14 \mathrm{~cm})$ and dumbbell shape (Table 2).

Postoperative complications occurred only in 1 male patient $(10 \%)$ in the anterior approach group and were Clavien-Dindo (CD) classification grade II (pelvic floor dyssynergia and lower limb weakness). The patient remained medicated without improvement. Complications occurred most frequently in the posterior approach group $(\mathrm{P}=0.021)$ in comparison to other approaches. Wound seroma and infection (CD classification grade II) were the most common (7 patients, 63.6\%), and all patients were managed with antibiotics and frequent dressing changes. One patient $(9.1 \%)$ had postoperative fluid collection in the right ischiorectal fossa (CD classification grade III) that was managed by pigtail tube drainage. No postoperative complications occurred in the combined approach group except in that 1 male patient (33.3\%) who had a posterior wound seroma and sexual dysfunction (CD classification grade II); he was under medical treatment. Postoperative pathology findings were benign for all except 2 male patients ( 1 with carcinoid tumor, 1 with adenocarcinoma). The first patient was managed with radiotherapy, and the other was referred to the medical oncology staff, who recommended close follow-up as a sufficient safety margin was obtained in the lower sacrectomy and coccygectomy via the combined approach.

Median mass size was $5.2 \mathrm{~cm}$ (range, 2.3-14 cm). Median follow-up was 12 months (range, 1-66 months), with no recurrence was observed. No postoperative mortality occurred.

Patients treated with the combined approach had a significantly larger cyst size $(\mathrm{P}<0.001)$, longer operation times $(\mathrm{P}<0.001)$, and more tumors located above the coccyx $(\mathrm{P}=0.002)$ than those treated using other approaches. No significant differences were noted for blood loss volume, time to first flatus, or tumor location relative to the pelvic floor muscles.

\section{DISCUSSION}

The incidence of retrorectal lesions is low in adults. A ratio of around 1/40,000 patients has been reported based on Mayo Clinic

Table 2. Comparison between the 3 surgical approaches

\begin{tabular}{|c|c|c|c|c|}
\hline Variable & Anterior approach $(n=10)$ & Posterior approach $(n=11)$ & Combined approach $(n=3)$ & P-value \\
\hline Coccyx & & & & 0.002 \\
\hline Above & $0(0)$ & $0(0)$ & $2(66.7)$ & \\
\hline Opposite & $3(30)$ & $2(18.2)$ & $1(33.3)$ & \\
\hline Below $^{a}$ & $7(70)$ & $9(81.8)$ & $0(0)$ & \\
\hline Pelvic floor & & & & 0.296 \\
\hline Supralevator $^{\mathrm{a}}$ & $4(40)$ & $1(9.1)$ & $0(0)$ & \\
\hline Supra- + infralevator & $6(60)$ & $9(81.8)$ & $3(100)$ & \\
\hline Infralevator & $0(0)$ & $1(9.1)$ & $0(0)$ & \\
\hline Size (cm) & $5.2 \pm 1.6$ & $4.3 \pm 1.3$ & $11.4 \pm 2.3$ & 0.001 \\
\hline $\mathrm{EBL}(\mathrm{mL})$ & $35(0-750)$ & $20(0-500)$ & $200(100-700)$ & 0.149 \\
\hline Operative time (min) & $157(100-360)$ & $90(45-200)$ & $320(315-400)$ & 0.001 \\
\hline Time to first flatus (day) & $1.8 \pm 1.1$ & $1.1 \pm 0.3$ & $2.0 \pm 1.00$ & 0.075 \\
\hline Clavien-Dindo classification grade & & & & 0.021 \\
\hline I & $0(0)$ & $0(0)$ & $1(33.3)$ & \\
\hline$\|$ & $1(10)$ & $7(63.6)$ & $0(0)$ & \\
\hline III & $0(0)$ & $1(9.1)$ & $0(0)$ & \\
\hline IV & $0(0)$ & $0(0)$ & $0(0)$ & \\
\hline V & $0(0)$ & $0(0)$ & $0(0)$ & \\
\hline
\end{tabular}

Values are presented as number (\%), mean \pm standard deviation, or median (range).

EBL, estimated blood loss.

aLocation of 3 cases of prerectal tumors. 


\section{Coloproctology Ahmad Sakr, et al.}

data [8]. Among retrorectal tumors, tailgut cysts arise from the remnants of the hind gut. Embryos normally form true tails, reaching maximum size at 35 days of gestation. The embryonic hindgut extends into the tail, forming the tailgut. By the week 8 of gestation, the tailgut normally regresses. Failure to obliterate this tailgut leads to the development of a tailgut cyst [9].

Tailgut cysts are rare and have a female predominance with a female to male ratio of $3: 1$ to $9: 1$. Patsouras and colleagues reported a female to male ratio of $7: 1$. Tailgut cysts commonly develop in the fifth decade of life; however, they can occur at any age [10-13]. In our study, the female to male ratio was $3: 1$ with a median patient age of 51.5 years.

Clinical presentation of tailgut cysts is usually nonspecific and misleading. Up to $50 \%$ of patients are asymptomatic, and cysts may be incidentally detected in radiological findings [13]. The other $50 \%$ of patients are symptomatic according to the largest reported case series of 53 patients by Hjermstad and Helwig [11]; symptoms usually occur from the local mass effect. Symptoms include rectal pain, constipation, obstructed defecation, tenesmus, painless rectal bleeding, dysuria, urinary frequency, lower abdominal pain, back pain, and lower limb neurological problems due to compression of the sacral plexus. Symptoms can also present as recurrent perianal suppuration, which is often misdiagnosed [11, 13]. In our series, $58.3 \%$ of patients were asymptomatic. Other patients' symptoms included lower abdominal pain, perineal pain, perianal swelling and fistula, and sacrococcygeal sinus.

One of the valuable diagnostic tools for detecting unilocular, multilocular, and small peripheral cysts is pelvic MRI [14]. Cysts usually have low signal intensity on T1-weighted images and high signal intensity on T2-weighted images. However, high signal intensity can also be seen on T1-weighted images because of the presence of mucinous material, high protein content, or hemorrhage into the cyst [15-17]. Thus, with multiple capabilities and good tissue contrast, MRI is superior for surgical planning for presacral masses. MRI also gives insights into whether cysts are malignant. When malignant, cysts appear heterogeneous with solid and cystic content and irregular borders. However, recurrent benign cysts may also appear this way $[14,15]$. In our study, all patients were diagnosed using MRI.

Controversy persists regarding preoperative biopsy. Hall et al. [18] demonstrated the possible use of ultrasound-guided needle biopsy as a diagnostic modality for tailgut cysts. Nevertheless, the use of this method is not advised as it carries the risk of seeding tumor cells [19]. Some patients experienced infection and tumor cell implantation when a biopsy sample was taken via transvaginal or transrectal (or, less commonly, para sacral) approaches. Some authors advised avoiding biopsy for masses that are purely cystic. For the cysts with mixed solid and cystic components, a percutaneous preoperative parasacral biopsy should be performed to determine treatment strategies with patients before surgery, optimize surgical plans, and inform patients about their prognosis and if postoperative adjuvant therapy is needed [12]. Others rec- ommend performing biopsies only when complete excision is impossible $[9,12]$. None of our patients underwent preoperative biopsy.

Tailgut cysts are usually lined with a variety of epithelia within the same cyst and may contain mucus; hence, malignant degeneration can occur within the cyst. Epithelial types are squamous, transitional, columnar, and cuboidal. Smooth muscle fibers are usually seen in association with cysts. Tailgut cyst may be associated with malignancy, mostly adenocarcinoma and carcinoid tumors. However, other reported tumors include neuroendocrine carcinoma, endometrioid carcinoma, adenosquamous carcinoma, squamous cell carcinoma, and sarcoma [11, 19, 20]. Our study had 2 patients with malignancy (both male: adenocarcinoma and carcinoid tumor). No females had malignant cysts. However, Mathis et al. [12] reported 4 female patients with malignancy (3 adenocarcinomas and 1 carcinoid tumor).

Studies reported a different incidence for malignant transformation of tailgut cysts. The rate of malignant transformation in the largest series in the literature in 1988 was 2\% [10]. Patsouras et al. [13] reported a $6 \%$ rate of malignant degeneration, other studies reported 13 to $40 \%[12,14]$, and in our study, the rate was $8 \%$.

Differential diagnosis should always be considered for retrorectal cysts. These are dermoid, epidermoid cyst, rectal duplication cyst, neuroenteric cyst, teratoma, anterior sacral meningocele, leiomyosarcoma, cystic lymphangioma, pyogenic abscess, neurogenic cyst, sacral chordoma, and tailgut cyst [19]. For prerectal cysts, the differential diagnosis includes prostatic utricle, simple cyst of the prostate, rectal duplication, simple cyst of the seminal vesicle, and bladder diverticulum [21].

The choice of surgical approach depends on several factors, including the degree of proximal extension of the cyst, cyst size, presence of infection, adherence to neighboring structures (bladder, ureters, rectum), and absence or presence of evidence of malignancy necessitating en bloc resection $[12,13]$. The posterior approach is most frequently described $[22,23]$.

For prerectal cysts, caution and careful dissection should be used to avoid injury to adjacent structures including the vagina, uterus, bladder, and rectum itself, especially when the cyst adheres to them. Injury occurred in our 3 prerectal cases with, 2 vaginal and 1 rectal. In our study, the risk of intra operative complications was high for prerectal compared to the retrorectal cysts.

Managing tailgut cysts via a trans rectal approach has been reported by some studies, most of which are case reports. McCarroll and Moore [24] reported managing a patient via a transanal minimally invasive surgery technique with no postoperative complications. Kildušis and Samalavičius [25] reported a patient managed by an open transrectal approach, also without complications. This study, noted that the transrectal approach can be for small noninfected low-lying cysts.

Debate persists about whether the coccyx should be removed with the cyst. Several studies advocate coccygectomy since it improves surgical exposure and decreases risk of recurrence assum- 
ing that the coccyx may harbor a nidus of totipotential cellular remnants that may cause recurrence $[4,26]$. However, the concern that the coccyx will increase risk of recurrence has not been demonstrated in other studies, especially for cysts not adherent to the coccyx and that can be removed entirely without coccygectomy; hence, coccyx removal is not required [27]. Removal of coccyx was based on the cyst itself and not the coccyx harboring aberrant remnants of the postanal gut leading to cyst formation. Recent trials report preserving the coccyx unless en bloc resection is required for malignancy or cysts densely adhere to the coccyx. Based on our findings, we removed the coccyx for 4 patients: 3 due to the cyst adhering to the coccyx and 1 for a large cyst requiring a combined coccygectomy and lower sacrectomy; the latter cyst was malignant.

We noted the importance of dissection in the proper surgical plane to avoid injury to the hypogastric nerves and pelvic plexuses during the anterior approach. Mathis et al. [12] reported complications of pelvic floor dyssynergia in 2 patients whose conditions improved with biofeedback therapy and 1 patient with sexual dysfunction after reoperation for recurrence. In our study, 1 patient had sexual dysfunction after the combined approach and 1 patient had pelvic floor dyssynergia and lower limb weakness after treatment with the anterior approach; both remain under medical treatment and follow-up. Other complications including postoperative wound seroma and infection were frequently observed after the posterior approach in our study; all were managed conservatively.

Avoiding injury to the rectal wall during dissection of the tailgut cyst is important using the posterior approach. Injury avoidance is facilitated by applying soft paraffin gauze roll packing in the rectum as a guide for rectum identification together with the preoperative bowel preparation.

Limited reports exist on recurrence rates after tailgut cyst resection. Some reported a range of $0 \%-16 \%$ and usually due to incomplete excision [13]. Another series found no recurrence among 12 patients after a median follow-up 54 months [3]. Mathias et al. [12] reported 1 case of recurrence after 5 years of followup. We had no cases of recurrence after a median follow-up of 12 months.

For surgical timing, some studies suggest routine follow-up for a symptomatic patient with low malignancy risk. may be appropriate. However, a definitive diagnosis cannot be obtained except after surgical excision and histopathological examination [28]. Accordingly, surgery is mandatory to exclude malignancy.

Despite being retrospective, our study included a large number of patients. The anterior approach and anterior part of the combined approach were performed laparoscopically in all cases, and no recurrence occurred after a median 12-month follow-up. We provide a management strategy according to tumor size and its correlation to pelvic floor muscles.

In conclusion, despite being uncommon, tailgut cysts can cause compression manifestations such as perineal or pelvic pain. Surgi- cal excision is mandatory regardless of symptom status because of the risk of malignancy. Complete surgical excision is a key treatment using an appropriate approach according to tumor size, location, and correlation with adjacent pelvic floor muscles.

\section{CONFLICTS OF INTEREST}

The authors report no potential conflicts of interest relevant to this article.

\section{REFERENCES}

1. Vega Menéndez D, Quintáns Rodríguez A, Hernández Granados P, Nevado Santos M, García Sabrido JL, Rueda Orgaz JA, et al. Tailgut cysts. Cir Esp 2008;83:53-60.

2. Dahan H, Arrivé L, Wendum D, Docou le Pointe H, Djouhri H, Tubiana JM. Retrorectal developmental cysts in adults: clinical and radiologic-histopathologic review, differential diagnosis, and treatment. Radiographics 2001;21:575-84.

3. Lev-Chelouche D, Gutman M, Goldman G, Even-Sapir E, Meller I, Issakov J, et al. Presacral tumors: a practical classification and treatment of a unique and heterogeneous group of diseases. Surgery 2003;133:473-8.

4. Wolpert A, Beer-Gabel M, Lifschitz O, Zbar AP. The management of presacral masses in the adult. Tech Coloproctol 2002;6:43-9.

5. Gips M, Melki Y, Wolloch Y. Cysts of the tailgut. Two cases. Eur J Surg 1994;160:459-60.

6. Sidoni A, Bucciarelli E. Ciliated cyst of the perineal skin. Am J Dermatopathol 1997;19:93-6.

7. Sung MT, Ko SF, Niu CK, Hsieh CS, Huang HY. Perirenal tailgut cyst (cystic hamartoma). J Pediatr Surg 2003;38:1404-6.

8. Jao SW, Beart RW Jr, Spencer RJ, Reiman HM, Ilstrup DM. Retrorectal tumors. Mayo Clinic experience, 1960-1979. Dis Colon Rectum 1985;28:644-52.

9. Prasad AR, Amin MB, Randolph TL, Lee CS, Ma CK. Retrorectal cystic hamartoma: report of 5 cases with malignancy arising in 2. Arch Pathol Lab Med 2000;124:725-9.

10. Killingsworth C, Gadacz TR. Tailgut cyst (retrorectal cystic hamartoma): report of a case and review of the literature. Am Surg 2005;71:666-73.

11. Hjermstad BM, Helwig EB. Tailgut cysts. Report of 53 cases. Am J Clin Pathol 1988;89:139-47.

12. Mathis KL, Dozois EJ, Grewal MS, Metzger P, Larson DW, Devine RM. Malignant risk and surgical outcomes of presacral tailgut cysts. Br J Surg 2010;97:575-9.

13. Patsouras D, Pawa N, Osmani H, Phillips RK. Management of tailgut cysts in a tertiary referral centre: a 10-year experience. Colorectal Dis 2015;17:724-9.

14. Yang DM, Park CH, Jin W, Chang SK, Kim JE, Choi SJ, et al. Tailgut cyst: MRI evaluation. AJR Am J Roentgenol 2005;184:1519-23.

15. Kim MJ, Kim WH, Kim NK, Yun MJ, Park YN, Lee JT, et al. Tailgut cyst: multilocular cystic appearance on MRI. J Comput Assist 


\section{Coloproctology $\begin{aligned} & \text { Annals of } \text { Single-center Exp } \\ & \text { Ahmad Sakr, et al. }\end{aligned}$}

Tomogr 1997;21:731-2.

16. Lim KE, Hsu WC, Wang CR. Tailgut cyst with malignancy: MR imaging findings. AJR Am J Roentgenol 1998;170:1488-90.

17. Moulopoulos LA, Karvouni E, Kehagias D, Dimopoulos MA, Gouliamos A, Vlahos L. MR imaging of complex tail-gut cysts. Clin Radiol 1999;54:118-22.

18. Hall DA, Pu RT, Pang Y. Diagnosis of foregut and tailgut cysts by endosonographically guided fine-needle aspiration. Diagn Cytopathol 2007;35:43-6.

19. Gönül II, Bağlan T, Pala I, Menteş B. Tailgut cysts: diagnostic challenge for both pathologists and clinicians. Int J Colorectal Dis 2007;22:1283-5.

20. Song DE, Park JK, Hur B, Ro JY. Carcinoid tumor arising in a tailgut cyst of the anorectal junction with distant metastasis: a case report and review of the literature. Arch Pathol Lab Med 2004;128: $578-80$

21. Jang SH, Jang KS, Song YS, Min KW, Han HX, Lee KG, et al. Unusual prerectal location of a tailgut cyst: a case report. World J Gastroenterol 2006;12:5081-3.
22. Hannon J, Subramony C, Scott-Conner CE. Benign retrorectal tumors in adults: the choice of operative approach. Am Surg 1994; 60:267-72.

23. Singer MA, Cintron JR, Martz JE, Schoetz DJ, Abcarian H. Retrorectal cyst: a rare tumor frequently misdiagnosed. J Am Coll Surg 2003;196:880-6.

24. McCarroll RH, Moore LJ. Transanal minimally invasive surgery for resection of retrorectal cyst. J Surg Case Rep 2018;2018:rjy021.

25. Kildušis E, Samalavičius NE. Surgical management of a retro-rectal cystic hamartoma (tailgut cyst) using a trans-rectal approach: a case report and review of the literature. J Med Case Rep 2014;8:11.

26. Izant RJ Jr, Filston HC. Sacrococcygeal teratomas. Analysis of forty-three cases. Am J Surg 1975;130:617-21.

27. Böhm B, Milsom JW, Fazio VW, Lavery IC, Church JM, Oakley JR. Our approach to the management of congenital presacral tumors in adults. Int J Colorectal Dis 1993;8:134-8.

28. Au E, Anderson O, Morgan B, Alarcon L, George ML. Tailgut cysts: report of two cases. Int J Colorectal Dis 2009;24:345-50. 